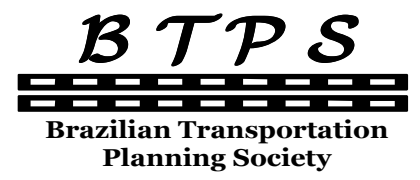
Journal of Transport Literature
Vol. 8, n. 2, pp. 295-324, Apr. 2014
Research Directory

\title{
Modelagem de redes logísticas com demandas sazonais: influência do custo de estoque e do crédito de ICMS
}

[Modeling of logistic networks with seasonality: influence of carrying cost and ICMS credit]

\author{
Ricardo Hamad*, Nicolau Dionísio Fares Gualda \\ University of São Paulo - Brazil, University of São Paulo - Brazil
}

Submitted 27 Apr 2013; received in revised form 11 Jul 2013; accepted 18 Sep 2013

\begin{abstract}
Resumo
Este artigo apresenta uma abordagem para localização de instalações em redes logísticas globais, com foco especial no tratamento dos estoques e impostos, aplicada em grandes empresas transnacionais do segmento agrícola operando no Brasil. Os resultados mostram que a restrição de capacidade de armazenagem e o custo de carregamento dos estoques, normalmente desprezados na maioria dos modelos, estão fortemente correlacionados com a definição do número de Centros de Distribuição. A metodologia, a partir da instalação de Centros de Distribuição em diferentes estados e/ou países do Mercosul, permitiu melhorias no fluxo de caixa da ordem de US\$ 22 milhões e mostrou o efeito negativo da legislação do ICMS que, para uma melhoria no fluxo de caixa, impõe um aumento nos custos logísticos. Mostra-se que, para a definição da rede logística em empresas credoras de ICMS, o modelo precisa contemplar pelo menos quatro elos da cadeia.
\end{abstract}

Palavras-Chave: rede logística, modelo de localização-estoque, planejamento tributário, crédito de ICMS, drawback.

\begin{abstract}
This paper presents an approach to solve the location problem in a global network with special focus on stocks and tax management. The methodology was applied successfully in large transnational agribusiness companies operating in Brazil. The results show that storage capacity constraints and inventory carrying costs, two components not usually included in location models, have a great correlation with the definition of the number of Distribution Centers. The methodology, from the installation of new facilities in different States or countries in MERCOSUR, improved the cash flow as much as US\$22 million and showed the negative effect of the ICMS legislation that drives to a logistics cost increase to improve the cash flow. It is demonstrated that the model must cover at least four echelons in the chain to solve the network problem of companies with credit of ICMS.
\end{abstract}

Key words: network design, inventory-location model, tax planning, ICMS tax, drawback.

* Email: ricardo.hamad@usp.br.

\section{Recommended Citation}

Hamad, R. and Gualda, N. D. F. (2014) Modelagem de redes logísticas com demandas sazonais: influência do custo de estoque e do crédito de ICMS. Journal of Transport Literature, vol. 8, n. 2, pp. 295-324.

- JTL/RELIT is a fully electronic, peer-reviewed, open access, international journal focused on emerging transport markets and published by BPTS - Brazilian Transport Planning Society. Website www.transport-literature.org. ISSN 2238-1031.

This paper is downloadable at www.transport-literature.org/open-access. 


\section{Introdução}

Este artigo propõe uma abordagem para a solução do problema de localização de instalações em nível global, sejam fábricas, fornecedores ou centros de distribuição, que amplia e melhora o modelo matemático apresentado por Hamad e Gualda (2006). O modelo incluiu fatores normalmente não cobertos por outros trabalhos, tais como, o crédito de ICMS, o benefício fiscal drawback, o custo do estoque total ao longo de uma rede logística global e restrições de armazenagem. A metodologia foi testada em grandes empresas com sérias dificuldades no gerenciamento de impostos e estoques.

A preocupação com a gestão de estoques começou a ganhar importância a partir da Revolução Industrial e tomou grande impulso após a Segunda Guerra Mundial. Conceitos como Just-InTime e Lean Manufacturing (Womack et al., 1990), a partir dos anos 70, demonstraram a importância da boa gestão dos estoques para o sucesso das empresas japonesas, o que contribuiu para que esse tema virasse foco de atenção em todo o mundo.

Bhutta (2001) afirma que, além dos estoques, dois outros fatores também entraram na lista de prioridades das empresas nas últimas décadas: a necessidade de se integrar ao mercado internacional (internacionalização) e a capacidade de adaptação às mudanças de mercado (resiliência). Segundo esse autor, uma cadeia de abastecimento eficaz envolve as definições do melhor nível de estoque em cada elo da cadeia e as definições sobre a melhor localização para as instalações e fornecedores. Bowersox e Closs (2001) argumentam que o interesse em ferramentas computacionais para melhorar a eficiência das decisões logísticas aumentou devido a avanços significativos na tecnologia de computação e foi influenciado pelo grande aumento da incerteza em relação à economia e às fontes de matérias primas. Atualmente, esta preocupação foi ampliada por pressões pelo crescimento sustentável, pela necessidade da conquista de novos mercados e pela competição agressiva principalmente de países da Ásia, com destaque para a China. 
A decisão sobre posicionamento dos estoques torna-se ainda mais complexa em empresas transnacionais. Depois da Segunda Guerra Mundial, com o fenômeno da globalização ganhando importância, muitas empresas se internacionalizaram visando à conquista de novos mercados e a racionalização de seus custos. As companhias brasileiras, de uma forma geral, não acompanharam essa tendência e só recentemente um maior número delas começou a abrir filiais ou fazer aquisições de empresas no exterior objetivando, além da expansão de suas vendas, a redução de seus custos de produção em países com condições mais competitivas. Esse interesse também foi impulsionado pelo benefício fiscal chamado drawback criado em novembro de 1966, através do qual as empresas exportadoras podem eliminar ou suspender os impostos sobre os insumos importados, uma vez comprovado que serão utilizados em produtos que foram ou serão exportados.

Outro fator importante que incentiva as empresas brasileiras a produzir em outros países é o chamado "Custo Brasil" que tem prejudicado o país na condição de fornecedor global de destaque, principalmente em itens de alto valor agregado como mostra estudo coordenado pela ABIMAQ (2010), entidade de classe dos fabricantes de equipamentos e máquinas. Esse estudo mostra que o preço dos produtos brasileiros, em média, é $36,3 \%$ maior do que os fabricados na Alemanha e nos EUA por conta de custos maiores e excesso de impostos. Além do excesso em número e alíquotas, a gestão dos impostos pelas empresas brasileiras se complica pela guerra fiscal entre os Estados causada pela intrincada legislação do Imposto sobre Circulação de Mercadorias (ICMS) que se diferencia em cada Estado.

Este artigo propõe, a partir de uma revisão da literatura (Seção 1) e dos impostos no Brasil (Seção 2), uma nova abordagem para o problema de localização de instalações em escala global (Seção 3) considerando o cálculo do custo do estoque total e o planejamento tributário, além de restrições de armazenagem normalmente não consideradas em outros modelos. A aplicação em quatro empresas transnacionais atuantes no Brasil é detalhada na Seção 4 e os resultados principais na Seção 5. 


\section{Modelos de localização e de estoques na literatura}

Modelos de localização têm sido estudados há mais de um século. A teoria da localização, incorporando elementos econômicos, é geralmente atribuída a Weber (1909), referência seminal para outros estudos. Recentemente, o desenvolvimento de modelos de localização global tem aumentado significativamente, mas a tendência para problemas de modelagem envolvendo apenas poucos elos da cadeia (dois ou três) é clara. Dogan e Goetschalckx (1999) apresentam um sistema de produção-distribuição multi-período com vendas sazonais para quatro elos da cadeia (fornecedor, primeiro e segundo estágios de produção e consumidor final), mas não incluem fatores usados em modelos globais, tais como impostos, inflação, taxas de câmbio e outros componentes que influenciam a definição dos países em que se quer atuar. Kurca e Koksalan (1996) são os primeiros a incluir a inflação como um fator a ser considerado e são também pioneiros na integração do planejamento financeiro e da produção. Programação Linear (LP) tem sido a ferramenta favorita para a solução de problemas de localização global e modelos discretos são os mais comuns (Bhutta, 2004). Snyder (2006) afirma que, devido à dificuldade para resolver problemas estocásticos, modelos de localização sob incerteza começaram a aparecer na literatura apenas a partir de 2000.

Love et al. (1988), Vidal e Goetschalckx (1997), Owen e Daskin (1998), Smits (2001), Revelle e Eiselt (2005) avaliaram uma variedade grande de modelos de localização. Bhutta (2004) apresentou uma avaliação de modelos de redes globais de distribuição e uma ampla revisão da literatura. Um resumo comparativo das características de modelos globais multifatores encontrados na literatura é apresentado na Tabela 1.

Apesar da preocupação com a gestão de estoques remontar aos primórdios da história humana (Garcia et al., 2006), modelos matemáticos criados para tal começaram a aparecer apenas no final do século XIX, mas sua associação com modelos de localização é bastante recente. Wilson (1934) explorou a fórmula desenvolvida por Harris (1913) para determinar a quantidade ideal do lote de compra ou produção utilizando o custo de estoques e o custo do processamento da ordem de produção/pedido de compra para itens que têm um padrão de demanda regular. Mas só no final do século passado autores começaram a utilizar o tamanho do lote para a determinação do custo de estoque em modelos de localização (Goetschalckx et alli, 1995 e Dogan e Goetschalckx, 1999). 
Tabela 1 - Comparação de modelos globais multi-fatores

\begin{tabular}{c|c|c|c|c|c|c|c|c|c|c|c|c}
\hline Autor & $\begin{array}{c}\text { Haug } \\
(1985)\end{array}$ & $\begin{array}{c}\text { Artnzen } \\
\text { et alli } \\
(1995)\end{array}$ & $\begin{array}{c}\text { Kirca } \\
\text { Kokasalan } \\
(1996)\end{array}$ & $\begin{array}{c}\text { Melachrinoudis } \\
(1996)\end{array}$ & $\begin{array}{c}\text { Mhumawala } \\
(1996)\end{array}$ & $\begin{array}{c}\text { Mohamed } \\
(1999)\end{array}$ & $\begin{array}{c}\text { Bhutta } \\
(2001)\end{array}$ & $\begin{array}{c}\text { Flipo } \\
(2000)\end{array}$ & $\begin{array}{c}\text { Hadjinicola } \\
\text { Kumar } \\
(2002)\end{array}$ & $\begin{array}{c}\text { Syam } \\
(2002)\end{array}$ & $\begin{array}{c}\text { Verter } \\
(2002)\end{array}$ & $\begin{array}{c}\text { Hamad } \\
\text { Gualda } \\
(2008)\end{array}$ \\
\hline $\begin{array}{c}\text { Tipo do } \\
\text { Modelo }\end{array}$ & PLIM & PLIM & PL & $\begin{array}{c}\text { Algoritmo } \\
\text { próprio }\end{array}$ & $\begin{array}{c}\text { PLIM } \\
\text { Binário }\end{array}$ & PLIM & PLIM & $\begin{array}{c}\text { Modela- } \\
\text { gem de } \\
\text { rede }\end{array}$ & $\begin{array}{c}\text { Progra- } \\
\text { mação por } \\
\text { metas }\end{array}$ & $\begin{array}{c}\text { PLIM } \\
\text { Binário }\end{array}$ & $\begin{array}{c}\text { Algoritm } \\
\text { o próprio }\end{array}$ & PLIM \\
\hline Objetivo & $\begin{array}{c}\text { Max. } \\
\text { Lucro }\end{array}$ & $\begin{array}{c}\text { Min. } \\
\text { Custo }\end{array}$ & $\begin{array}{c}\text { Max. } \\
\text { Lucro }\end{array}$ & $\begin{array}{c}\text { Max. } \\
\text { Lucro }\end{array}$ & $\begin{array}{c}\text { Max. } \\
\text { Lucro }\end{array}$ & $\begin{array}{c}\text { Min. } \\
\text { Custo }\end{array}$ & $\begin{array}{c}\text { Max. } \\
\text { Lucro }\end{array}$ & $\begin{array}{c}\text { Min. } \\
\text { Custo }\end{array}$ & $\begin{array}{c}\text { Max. } \\
\text { Lucro }\end{array}$ & $\begin{array}{c}\text { Min. } \\
\text { Custo }\end{array}$ & $\begin{array}{c}\text { Min. } \\
\text { Custo }\end{array}$ & $\begin{array}{c}\text { Min. } \\
\text { Custo }\end{array}$ \\
\hline Períodos & 2 & 18 & 12 & 3 & 4 & 3 & 3 & 12 & 1 & 1 & 1 & 1 \\
\hline Instalações & 2 & 33 & 1 & 5 & 5 & 2 & 3 & 10 & 3 & 3 & 13 & 17 \\
\hline Mercados & 1 & & 1 & 6 & 3 & 4 & & & 2 & 49 & 27 \\
\hline Produtos & 1 & & 1 & 1 & 1 & 1 & 2 & 16 & 1 & 1 & 1 & 4 \\
\hline
\end{tabular}

Fontes: Autores e Bhutta (2004)

Womack et al. (1990) afirmam que o excesso de estoque tornou-se uma das principais causas de desperdício em empresas, pois é usado para mascarar atividades ineficientes. Hines e Taylor (2000) em sua obra clássica sobre a implantação do lean manufacturing em empresas, mostram que indústrias que não são consideradas de classe mundial desenvolvem, em média, apenas 5\% de atividades que agregam valor aos seus clientes. $35 \%$ de atividades não agregam valor aos clientes, mas agregam valor ao negócio e, portanto, são justificadas. As $60 \%$ restantes não agregam valor nem ao cliente, nem ao negócio e, portanto, são atividades descartáveis que, se eliminadas, reduziriam os excessos de estoque. Não existem dados precisos sobre a realidade atual no Brasil, mas vários autores, tais como, Machado (2006) afirmam que a improdutividade com consequente geração de desperdícios é clara nas empresas brasileiras, e isso já começa no desenvolvimento de novos produtos. Crimson \& Co, a maior consultoria de Supply Chain do Reino Unido, em novembro de 2011 coordenou entrevistas sobre gestão da cadeia com 300 líderes em 200 empresas de vários segmentos 
(Scarpati, 2011). O gerenciamento dos estoques situou-se entre as maiores preocupações, seguido do gerenciamento da demanda e da otimização da rede logística.

Pesquisa realizada pela consultoria Price Waterhouse Coopers (PwC, 2011) com líderes empresariais em todo o mundo traz resultados semelhantes, tais como, busca por novas alternativas de expansão dos negócios, disciplina na gestão de custos e riscos, e capacidade de entender as mudanças estruturais na economia mundial e responder a elas com um melhor posicionamento dos estoques, instalações e recursos. Nessa pesquisa, líderes brasileiros também mostraram sua grande preocupação com estoques, já que estes representam, em média, mais de 50\% do ativo fixo das empresas no Brasil (Christopher, 2002). Devido a esta reconhecida importância, a inclusão dos custos de estoque em modelos de localização tem-se tornado mais recorrente como em Flipo (2000), Bhutta (2001), Smits (2001), Nozick e Turnquist (2001), Flipo \& Finke (2001), Syam (2002), Goetschalckx et al. (2002), Bhutta et al. (2003), Shen et al. (2003), Hale et al. (2008). Croxton e Zinn (2005) dão uma importante contribuição para a análise dos estoques na definição de uma malha logística e parte da sua formulação é usada neste trabalho.

De acordo com Shen (2007), modelos para gestão de estoques tendem a ignorar a decisão de localização e seus custos associados, enquanto modelos de localização tendem a ignorar os custos de estoque e operacionais relacionados com a falta de estoque, bem como a incerteza da demanda e dos efeitos que as políticas de reposição têm nos custos de estoque e entrega. Uma das razões para esta desconexão é a falta de informações operacionais detalhadas durante a fase estratégica do projeto, enquanto que as decisões sobre a localização das instalações são feitas geralmente sem dados adequados de estoque e custos de distribuição. Outro problema apontado por Shen é a consideração de que a capacidade é ilimitada para cada Centro de Distribuição (CD). Em Shen et al. (2003), um modelo de localização-estoque é proposto buscando preencher esta lacuna, mas o modelo considera um único fornecedor e apenas CDs para a armazenagem de produtos acabados.

Smits (2001) propõe um modelo de rede que calcula os custos de estoque a partir do nível de estoque médio dado pela taxa de ordens de chegada, taxa de entrega e tamanho do lote de entrega, mas inclui apenas produtos acabados. A falta de consideração de outros tipos de estoque, tais como matérias primas, também ocorre na maioria dos modelos, tal como o proposto por Syam (2002), no qual o material é calculado usando o ciclo de vida da ordem e 
do fluxo de materiais em cada arco durante vários períodos. Em Hadjinicola e Kumar (2002), o estoque médio é aproximado por metade da demanda e, em Gross et al. (1981), o nível de estoque médio e a falta de estoque são calculados usando o modelo de Clark (1958).

Apenas alguns modelos integrados de localização de estoque, tais como Arntzen et al. (1995), Goetschalckx et al. (1995), Dogan e Goetschalckx (1999) e Smits (2001), consideram os estoques em trânsito, mas apenas o estoque de produtos acabados que estão sendo transferidos. Isso faz com que matérias-primas em trânsito, parte representativa dos estoques em empresas globais com materiais importados por via marítima, não sejam consideradas.

Shapiro (2001) aponta como incorporar modelos de gestão de estoque para a definição estratégica de redes logísticas e afirma que em modelos dinâmicos (modelos que consideram alterações em parâmetros como custo e capacidades ao longo do tempo), a consideração comum de que o estoque no fim do período é zero não é uma abordagem correta, porque haverá produção e distribuição nos períodos seguintes. Além disso, se o tempo $t$ é muito longo (um mês ou mais), modelos dinâmicos que usam o estoque no fim do período para o cálculo do custo de carregamento de estoques, tais como em Mohamed (1999), Dogan e Goettschalckx (1999) e Arntzen et al. (1995), podem subavaliar os estoques, já que o estoque no fim do período tende a ser menor do que o estoque médio durante o período. Duas características principais explicam a importância de modelos dinâmicos em modelos de localização, especialmente para decisões táticas: muitas vezes há mudança nos custos ao longo do tempo e também pode haver custos significativos para a transferência e/ou dimensionamento de novas plantas (Erlenkotter, 1981). Mas, para fins estratégicos, modelos estáticos de localização são bastante apropriados (Ballou, 1998).

$\mathrm{Na}$ maioria dos modelos de localização estudados, os estoques de matérias-primas, manutenção e outros não são considerados. Na modelagem de uma fábrica, isso pode significar deixar de lado entre 30 e $70 \%$ dos estoques. Nas quatro empresas participantes deste estudo, o valor desse tipo de estoque ficou em torno de 40 a $60 \%$ do total geral. Richardson (1995) apresenta um estudo para os EUA que mostra um custo financeiro entre $6 \%$ e $12 \%$ e um custo total de $25 \%$ a $55 \%$. Estima-se que o custo financeiro é ainda maior no Brasil.

Smits (2001) afirma que o custo de estoque tem sido aplicado em modelos de localização apenas mais recentemente. Uma explicação para esse fenômeno é a "regra da raiz quadrada", 
que faz com que a relação entre estoque de segurança e o número de centros de distribuição não seja linear (Maister, 1976). Os modeladores que preferem desenvolver soluções usando programação linear acabam por neglicenciar os estoques e seus custos ou simplificam o problema. Nozick e Turnquist (2001), por exemplo, usam a regra de raiz quadrada para o cálculo do custo de estoque considerando que para $s \geq 15$ ( $s=\mathrm{n}^{\circ}$ de $\mathrm{CDs}$ ), a função raiz quadrada converge para uma função linear.

Em problemas de localização de instalações, especialmente em escala global, há necessidade de incluir alguns outros fatores que afetam a tomada de decisão, tais como a flutuação das taxas de câmbio, os custos de capital, as variações nas taxas de juros e de inflação. Modelos criados para as corporações transnacionais ganharam importância nos últimos anos (Bhutta, 2004) e o impacto dos impostos sobre a rede de logística começou a ter seu destaque. Yoshizaki (2002), a partir da aplicação de um modelo de três elos, comprova a importância da consideração de impostos na definição de uma rede logística em empresas brasileiras. Arntzen et al. (1995) também demonstram a importância dos impostos para a definição da localização de instalações em escala global.

\section{Impostos no Brasil}

Há três bases clássicas de tributação no Brasil: propriedade, renda e consumo, além dos reguladores, como os relacionados a comércio exterior (impostos de importação e de exportação). Esses impostos, conforme dados oficiais do Ministério da Fazenda de 2012, representam uma carga tributária de 36,3\% em relação ao PIB, um dos maiores do mundo e comparável apenas com as cargas tributárias dos países europeus. Iniciativas mais concretas para desoneração das empresas têm acontecido apenas recentemente. Dos 86 impostos, tarifas e taxas que são aplicados sobre as vendas, ativos e renda existentes no Brasil, o Imposto de Importação (II) e o Imposto sobre Circulação de Mercadorias e Serviços (ICMS) são os que dependem fortemente da localização das fábricas, fornecedores e área de cobertura dos centros de distribuição.

O II é um imposto federal criado para gerir a entrada de mercadorias no mercado interno e tem sido usado como uma ferramenta para negociações econômicas com outros países e como uma barreira para proteger a indústria nacional. O benefício fiscal Drawback foi criado para 
incentivar as exportações e tem duas modalidades principais: o de suspensão (o imposto de importação não incide na entrada dos insumos em quantidade e qualidade equivalentes ao produto a ser exportado) e o de isenção (o imposto incide inicialmente sobre o valor do insumo para, depois de transformada em produto acabado e exportado, transformar-se em um crédito a ser usado no momento da importação para reposição desse item).

O ICMS é um Imposto de Valor Agregado (IVA) estadual que incide quando há circulação de mercadorias ou quando há o pagamento de serviços de comunicação, transporte intermunicipal e interestadual, e desembaraço aduaneiro de mercadoria importada. Este imposto é cobrado no Estado de origem das mercadorias ou serviços, exceto no caso do petróleo, eletricidade e seus derivados, onde o imposto é cobrado no estado de destino. As mercadorias transportadas entre estabelecimentos de uma mesma empresa são tributadas de acordo com o preço de transferência declarado na nota fiscal, valor que normalmente representa o Custo dos Produtos Vendidos (CPV), enquanto as vendas a terceiros são tributadas pelo preço de venda indicado na fatura. O CPV é calculado através da soma do estoque inicial e do custo de produção (insumos + mão de obra direta e indireta aplicados na produção dos bens vendidos + gastos gerais de fabricação) subtraídos do inventário final. Cada transação gera débitos no Estado de origem e créditos no Estado de destino. As taxas para transações entre estados e dentro do mesmo estado variam significativamente, bem como a base de sua tributação. Devido às suas características regionais, as alíquotas são diferentes para as operações intra e interestadual e o balanço entre débitos e créditos deve ser calculado para cada um dos estados brasileiros onde as matérias-primas e produtos acabados foram adquiridos, transferidos e/ou vendidos. Um débito gerado em um elemento ou elo da cadeia torna-se um crédito no elo seguinte. Quando a empresa gera um crédito de ICMS e não tem condições de usá-lo, o total de créditos não utilizados deve ser considerado como um valor negativo no balanço da empresa e é comumente chamado de "crédito morto" ou "crédito podre". Exemplificando: uma empresa do ramo agrícola que fabrica seus produtos em São Paulo e os transfere para um CD em outro Estado paga um valor de ICMS no Estado de origem que se torna um crédito no Estado de destino. Se, por conta de benefícios fiscais como, por exemplo, isenção do ICMS para produtos agrícolas vendidos no Estado (Tomich et alli, 1998) ou isenção na exportação de produtos acabados (Lei Kandir de Setembro de 1996), esse crédito se torna sempre crescente e inutilizável gerando, dessa forma, um "crédito morto" 
que precisará ser contabilizado como uma despesa a partir do reconhecimento da impossibilidade de sua utilização.

\section{A metodologia e o modelo matemático}

O modelo desenvolvido supõe uma empresa contribuinte do ICMS, sem o direito de usos alternativos dos créditos de ICMS. A principal preocupação durante o desenvolvimento da abordagem e do modelo matemático foi torná-lo fácil de ser compreendido e adotado pelas empresas. Em relação ao modelo apresentado por Hamad e Gualda (2006), citam-se as seguintes melhorias:

- Custos de estoques calculados de acordo com Hamad e Gualda (2011);

- Tratamento para o cálculo dos volumes e custo do estoque de segurança nos CDs;

- Desenvolvimento de um guia para cálculo do custo de carregamento dos estoques;

- Desenvolvimento de uma concorrência nacional para seleção de parceiros de transporte e armazenagem;

- Inclusão de forma segregada dos custos de manuseio nos CDs secundários;

- Inclusão de forma segregada dos custos de armazenagem nos CDs secundários;

- Inclusão do índice de sazonalidade para verificação das restrições ligadas às capacidades dos CDs primários e secundários;

- Inclusão do ad-valorem nos custos de transporte;

- Inclusão do estoque inicial nos CDs;

- Segregação das análises referentes a impostos;

- Inclusão de restrições ligadas à geração do crédito podre (a serem aplicadas ad hoc);

- Análise de cenários usando o conceito de Fluxo de Caixa Logístico (fluxo de saída de caixa considerando os custos logísticos e o aproveitamento dos créditos de ICMS).

O objetivo do modelo é a minimização de custos. Por premissa, a demanda não é impactada pelo desenho da rede. Como se supõe que todas as demandas serão atendidas, o custo das vendas perdidas devido à falta de estoque é desconsiderado. Volumes e capacidades foram convertidos para uma unidade padrão denominada tonelada-equivalente. O modelo será detalhado considerando apenas quatro elos na cadeia, já que isso atende à grande maioria dos problemas desse tipo, mas, caso necessário, o modelo pode ser facilmente estendido para 
cinco ou mais elos. O primeiro elo representa o fornecedor do produto acabado, que pode ser uma fábrica própria ou um fornecedor de produtos para revenda. O segundo elo da cadeia é o $\mathrm{CD}$ principal que, normalmente, fica conjugado à fábrica, mas que pode representar um armazém para estocagem de produtos de revenda. $\mathrm{O}$ terceiro elo representa o $\mathrm{CD}$ secundário a partir de onde os produtos acabados serão enviados para o mercado consumidor. Dependendo do problema, o modelo também pode ser adaptado para que o primeiro elo seja um fornecedor de matéria prima principal (ingrediente ativo, por exemplo, no caso de indústria química) e o segundo elo, as fábricas de produto acabado.

Para calcular o estoque anual médio de um dado elo $f$ em volume $\left(\mathrm{AIV}_{f}\right)$ e valor financeiro $\left(\mathrm{AIS}_{f}\right.$ ), adotou-se o modelo de Hamad e Gualda (2011) multiplicando-se o CPV pelo valor esperado da cobertura do estoque em dias, da fábrica ou do CD. A cobertura é o inverso do giro de estoque e é um indicador clássico adotado pela maioria das empresas para demonstrar a eficácia na gestão de seus estoques. A popularidade deste indicador vem de sua simplicidade e da disponibilidade imediata dos dados já que os estoques são, devido a exigências legais e práticas de negócio, controlados de forma rotineira pelas empresas (Ballou, 1998). O valor da cobertura é geralmente chamado DOH (Days-On-Hand) e, neste estudo, será separado em DOHI (calculado a partir do estoque em R\$) e DOHS (calculado a partir do estoque em tonelada-equivalente). DOHI de um elo $f$ é calculado pela divisão entre valor do estoque médio anual (AIS) e o CPV para fabricar os vários produtos $\boldsymbol{m}$ :

$$
D O H I_{f}=\frac{A I S_{f}}{\sum_{m} C P V_{m f}} \quad 365
$$

ou,

$$
A I S_{f}=\frac{\mathrm{DOHI}_{f} \sum_{m} C P V_{m f}}{365}, \quad \forall f
$$

onde:

$\mathrm{DOHI}_{f}=$ cobertura esperada (baseado em $\mathrm{R} \$$ ) para o elo $f$, em dias, que pode ser calculada a partir de bases históricas ou por comparação com outras instalações similares. 
Para novas instalações, o DOH pode ser estimado considerando o seguinte:

- Lead-time do material em trânsito para um elo específico: quanto maior o tempo de trânsito, maior a cobertura;

- Qualidade do processo de planejamento: quanto melhor a precisão nas previsões de venda, a qualificação dos planejadores, a flexibilidade na aquisição de recursos e a implantação de boas práticas no processo de planejamento, menor a cobertura;

- Sazonalidade: quanto maior o índice de sazonalidade, maior a cobertura;

- Confiabilidade de fornecedores: este determina diretamente o tamanho dos estoques de segurança e, consequentemente, o nível de DOH.

Dependendo do segmento de negócio, da maturidade dos produtos e do conhecimento do mercado, o DOH pode ser adotado por similaridade com empresas semelhantes, a partir das informações de giro de estoque disponibilizadas geralmente no balanço ou em relatórios anuais dessas empresas. Há também sites na Internet que fornecem informações financeiras e estatísticas sobre estoques e CPV das empresas, que também podem ser fontes de dados para estimar o DOH.

O custo de oportunidade financeira $(\mathrm{CI})$ é dado pela seguinte fórmula:

$$
C I_{f}=A I S_{f} x C F
$$

Onde:

CF é o Custo de capital da Companhia, ou custo de oportunidade financeira, em \% do valor do estoque total. $\mathrm{O}$ custo de capital é muito significativo no Brasil, devido à alta taxa básica de juros definida pelo Banco Central. Nas quatro empresas transnacionais que participaram deste estudo, esse custo ficou entre $8 \%$ e $15 \%$.

O conceito de DOHE, cobertura em número de dias com base no volume transportado, é utilizado para um determinado item ou grupo de itens. Ele considera uma unidade de volume padrão e calcula $A I V_{f}$ usando o mesmo raciocínio utilizado para $A I S_{f}$ : 


$$
A I V_{f}=\frac{\operatorname{DOHE}_{f} \sum_{m} D s t r_{m f}}{365}, \forall f
$$

onde:

$\mathrm{AIV}_{f}=$ Estoque médio anual em volume no elo $\boldsymbol{f}$ $\mathrm{DOHE}_{f}=$ cobertura esperada em volume no elo $\boldsymbol{f}$ em número de dias $\sum_{m} D s t r_{m f}=$ Volume total do Produto $\boldsymbol{m}$ distribuído para o mercado $\boldsymbol{f}$

DOHE pode ser estimado com base em dados históricos ou por comparação com outras empresas semelhantes.

Os dados foram coletados junto á área financeira das empresas e a partir de uma concorrência para avaliar custos fixos e variáveis de transporte, manuseio e armazenagem em diferentes CDs. Todos os dados e os resultados foram validados pelas empresas.

Embora o modelo proposto possa ser aplicado em cadeias logísticas com um número ilimitado de elos (fornecedores, fábricas, etc.), neste trabalho definiu-se uma rede logística com quatro elos (fábricas, CDs principais, CDs secundários e mercado consumidor). Os índices usados foram os seguintes:

(i) - fábricas próprias ou de terceiros (primeiro elo da cadeia)

(j) - CDs principais (segundo elo)

(k) - CDs secundários (terceiro elo)

(I) - regiões de venda (quarto elo)

(m) - famílias de produtos

(n) - estados brasileiros onde as mercadorias são entregues ou fabricadas

As Variáveis de Decisão são representadas pelos volumes do produto $\boldsymbol{m}$ transferidos de $\boldsymbol{i}$ para $\boldsymbol{j}$ com Drawback (TrnsC $\left.\mathrm{mij}_{\mathrm{ij}}\right)$ ou sem ele $\left(\operatorname{Trns}_{\mathrm{mij}}\right)$, entre $\boldsymbol{j}$ e $\boldsymbol{k}\left(\operatorname{Trns}_{\mathrm{mjk}}\right)$ e entre $\boldsymbol{k}$ e $\boldsymbol{l}\left(\right.$ Dstr $\left._{\mathrm{mkl}}\right)$, todas em tonelada-equivalente. Existem ainda variáveis binárias para $\mathrm{CDs}$ primários e secundários $\left(\mathrm{Z}_{\mathrm{jm}}\right.$ e $\left.\mathrm{Z}_{\mathrm{k}}\right)$. 
Os Parâmetros utilizados no modelo são os seguintes:

$\mathrm{DEM}_{\mathrm{ml}}=$ demanda do produto $\boldsymbol{m}$ no mercado $\boldsymbol{l}$

$\mathrm{CAPP}_{\mathrm{mi}}=$ capacidade de produção de $\boldsymbol{m}$ na fábrica ou fornecedor $\boldsymbol{i}$

$\mathrm{CAPH}_{\mathrm{mj}}=$ capacidade de movimentação no $\mathrm{CD} \boldsymbol{j}$ reservados para o produto $\boldsymbol{m}$

CAPS $_{\mathrm{j}}=$ capacidade de armazenamento do $\mathrm{CD} j$

$\mathrm{CAPH}_{\mathrm{km}}=$ capacidade de movimentação do CD $\boldsymbol{k}$ reservado para o produto $\boldsymbol{m}$

$\mathrm{CAPS}_{\mathrm{k}}=$ capacidade de armazenamento do CD $\mathbf{k}$

$\mathrm{MINP}_{\mathrm{mi}}=$ produção mínima de $\boldsymbol{m}$ na fábrica $\boldsymbol{i}$

$\mathrm{MINH}_{\mathrm{mj}}=$ manuseio mínimo de $\boldsymbol{m}$ no CD $\boldsymbol{j}$

$\mathrm{MINH}_{\mathrm{mk}}=$ manuseio mínimo de $\boldsymbol{m}$ no CD $\boldsymbol{k}$

$\mathrm{EICD}_{\mathrm{mk}}=$ Estoque inicial de $\boldsymbol{m}$ no CD $\boldsymbol{k}$

$\mathrm{CUST}_{\text {mij }}=$ Custo unitário (R $\$$ /unidade padrão) para transferir $\boldsymbol{m}$ de $\boldsymbol{i}$ para $\boldsymbol{j}$

$\mathrm{CUST}_{\mathrm{mjk}}=$ Custo unitário (R $\$$ /unidade padrão) para transferir $\boldsymbol{m}$ de $\boldsymbol{j}$ para $\boldsymbol{k}$

$\mathrm{CUSD}_{\mathrm{mkl}}=$ Custo unitário $(\mathrm{R} \$$ /unidade padrão) para distribuir $\boldsymbol{m}$ de $\boldsymbol{k}$ para $\boldsymbol{l}$

$\mathrm{CUSBC}_{\mathrm{mi}}=$ Custo unitário (R\$/unidade padrão) de $\boldsymbol{m}$ em $\boldsymbol{i}$ com o benefício de Drawback

CUSBS $_{\mathrm{mi}}=$ Custo unitário (R $\$$ /unidade padrão) de $\boldsymbol{m}$ em $\boldsymbol{i}$ sem o benefício de Drawback

$\mathrm{CUSH}_{\mathrm{mj}}=$ Custo de manuseio $(\mathrm{R} \$ /$ unidade padrão) de $\boldsymbol{m}$ no $\mathrm{CD} \boldsymbol{j}$

$\mathrm{CUSH}_{\mathrm{mk}}=$ Custo de manuseio (R\$/unidade padrão) de $\boldsymbol{m}$ no CD $\boldsymbol{k}$

$\mathrm{CUSS}_{\mathrm{j}}=$ Custo de armazenagem (R $\$$ /unidade padrão) em $\boldsymbol{j}$

$\mathrm{CUSS}_{\mathrm{k}}=$ Custo de armazenagem (R\$/unidade padrão) em $\boldsymbol{k}$

CUSFix $_{j m}=$ Custo fixo $(\mathrm{R} \$)$ no $\mathrm{CD} \boldsymbol{j}$ para manusear $\boldsymbol{m}$

CUSFix $_{\mathrm{k}}=$ Custo fixo $(\mathrm{R} \$)$ no CD $\boldsymbol{k}$

CUSAV $_{\mathrm{ij}}=A d$-valorem $(\%$ do valor das mercadorias transportadas) de $\boldsymbol{i}$ para $\boldsymbol{j}$

$\operatorname{CUSAV}_{\mathrm{jk}}=A d$-valorem $(\%$ do valor das mercadorias transportadas) de $\boldsymbol{j}$ para $\boldsymbol{k}$

CUSAV $_{\mathrm{kl}}=A d$-valorem (\% do valor de mercadorias transportadas) de $\boldsymbol{k}$ para $\boldsymbol{l}$

$\mathrm{ICMS}_{\mathrm{ij}}=\operatorname{ICMS}$ (\% sobre o preço de transferência dos bens) de $\boldsymbol{i}$ para $\boldsymbol{j}$. Se o elo $\boldsymbol{i}$ é fora do país, a taxa de ICMS a ser aplicada é a do porto de entrada no Brasil

$\mathrm{ICMS}_{\mathrm{jk}}=\operatorname{ICMS}(\%$ sobre o preço de transferência de bens finais) de $\boldsymbol{j}$ para $\boldsymbol{k}$

$\mathrm{ICMS}_{\mathrm{kl}}=\mathrm{ICMS}$ (\% sobre o preço de venda de bens finais) de $\boldsymbol{k}$ para $\boldsymbol{l}$

$\mathrm{PI}_{\mathrm{m}}=$ Preço de transferência ( $\mathrm{R} \$$ /unidade padrão) de $\boldsymbol{m}$ - definido pela área financeira, este preço é normalmente o CPV do produto $\boldsymbol{m}$

$\mathrm{PF}_{\mathrm{mkl}}=$ preço de vendas $(\mathrm{R} \$ /$ unidade padrão) do produto $\boldsymbol{m}$ entregues em $\boldsymbol{l}$ a partir de $\boldsymbol{k}$ 
$\mathrm{P}_{\mathrm{j}}=$ índice de sazonalidade (\%) para $\boldsymbol{j}$ - usado para calcular o volume dos estoques no pico $\mathrm{P}_{\mathrm{k}}=$ índice de sazonalidade $(\%)$ para $\boldsymbol{k}$ - usado para calcular o volume dos estoques no pico

A função objetivo inclui custos fixos e variáveis e foi adaptada conforme o cenário estudado.

A Função Objetivo para um dos cenários foi minimizar Custo de Operações (CO),

$$
\begin{aligned}
& C O=\sum_{m} \sum_{i} \sum_{j}\left(\left(C U S T_{m i j}+C U S B C_{m i}+C U S H_{m j}+C U S A V_{i j} \cdot P I_{m}\right) \cdot \operatorname{Trns} C_{m i j}\right) \rightarrow(A) \\
& +\sum_{m} \sum_{i} \sum_{j}\left(\left(C U S T_{m i j}+C U S B S_{m i}+C U S H_{m j}+C U S A V_{i j} \cdot P I_{m}\right) \cdot T r n s S_{m i j}\right) \rightarrow(B) \\
& +\sum_{m} \sum_{j} \sum_{k}\left(C U S T_{m j k}+C U S H_{m k}+C U S A V_{j k} \cdot P I_{m}\right) \cdot T r n s_{m j k} \rightarrow(C) \\
& +\sum_{m} \sum_{k} \sum_{l}\left[\left(C U S D_{m k l}+C U S A V_{k l} \cdot P F_{m k l}\right) \cdot D s t r_{m k l}\right]+C U S E S \rightarrow(D) \\
& +\sum_{j}\left(\text { CUSFix }_{j m} \cdot Z_{j m}\right)+\sum_{k}\left(\text { CUSFix }_{k} \cdot Z_{k}\right) \rightarrow(E) \\
& +\sum_{i} C I_{i}+\sum_{j} C I_{j}+\sum_{k} C I_{k} \rightarrow(F) \\
& +\sum_{m} \sum_{j} \sum_{k}\left(\text { CUSS }_{j} \cdot\left(\left(\text { DOHE }_{j} \cdot \text { Trns }_{m j k}\right) / 365\right)\right)+\sum_{m} \sum_{k} \sum_{l}\left(\text { CUSS }_{k} \cdot\left(\left(\text { DOHE }_{k} \cdot \text { Dstr }_{m k l}\right) / 365\right)\right) \rightarrow(G)
\end{aligned}
$$

Onde:
(A) - Custos de transferência do produto acabado com o benefício de Drawback de $\boldsymbol{i}$ para $\boldsymbol{j}$
(B) - Custos de transferência do produto acabado sem o benefício de Drawback de $\boldsymbol{i}$ para $\boldsymbol{j}$
(C) - Custos de transferência do elo $\boldsymbol{j}$ para o elo $\boldsymbol{k}$
(D) - Custo de distribuição de $\boldsymbol{k}$ para $\boldsymbol{l}+$ Custo do estoque de Segurança (vide equação (24))
(E) - Total de custos fixos nos CDs
(F) - Custo de carregamento dos estoques em $\boldsymbol{i}, \boldsymbol{j}$ e $\boldsymbol{k}$ - conforme equação (3)
(G) - Custos de armazenagem nos CDs $\boldsymbol{j}$ e $\boldsymbol{k}$

Além da não negatividade, temos, no modelo, as seguintes Restrições:

Demanda a ser integralmente atendida:

$$
D E M_{m l} \leq \sum_{k} D_{s t r_{m k l}} \quad \forall l, m
$$


Limitações da capacidade produtiva de $\boldsymbol{m}$ no fornecedor ou unidade industrial $\boldsymbol{i}$ :

$$
D E M_{m l} \leq \sum_{k} \text { Dstr }_{m k l} \quad \forall l, m
$$

Limitações da capacidade produtiva de $\boldsymbol{m}$ no fornecedor ou unidade industrial $\boldsymbol{i}$ :

$$
\sum_{j}\left(\operatorname{Trns}_{m i j}+\operatorname{Trns}_{m i j}\right) \leq C A P P_{m i} \quad \forall m, i .
$$

Produção mínima de $\boldsymbol{m}$ no fornecedor ou unidade industrial $\boldsymbol{i}$ :

$$
\sum_{j}\left(\operatorname{Trns} C_{m i j}+\operatorname{Trns}_{m i j}\right) \geq M I N P_{m i} \quad \forall m, i .
$$

Capacidade de manuseio no $\mathrm{CD} j$ para o produto $\boldsymbol{m}$ :

$$
D E M_{m l} \leq \sum_{k} D_{s t r_{m l}} \quad \forall l, m
$$

Manuseio mínimo permitido no $\mathrm{CD} \boldsymbol{j}$ do produto $\boldsymbol{m}$ :

$$
\sum_{k} \operatorname{Trns}_{m j k} \geq \operatorname{MinH}_{m j} . Z_{j m} \quad \forall j, m
$$

Capacidade de armazenagem no $\mathrm{CD} j$ :

$$
A I V_{j} x P_{j} \leq C A P S_{j} \quad \forall j .
$$

Capacidade de manuseio no CD $\boldsymbol{k}$ :

$$
\sum_{l} \text { Dstr }_{m k l} \leq \mathrm{CAPH}_{k m} Z_{k} \quad \forall k, m
$$


Capacidade de armazenagem no CD $\boldsymbol{k}$ :

$$
A I V_{k} x P_{k} \leq C A P S_{k} \quad \forall k .
$$

Manuseio mínimo permitido no CD $\boldsymbol{k}$ do produto $\boldsymbol{m}$ :

$$
\sum_{l} D_{s t r_{m k l}} \geq \operatorname{MINH}_{m k} Z_{k} \quad \forall k, m .
$$

Balanço de massa do produto $\boldsymbol{m}$ no $\mathrm{CD} j$ :

$$
\sum_{i}\left(\operatorname{Trns}_{m i j}+\operatorname{Trns} S_{m i j}\right)=\sum_{k} \operatorname{Trn} s_{m j k} \quad \forall m, j .
$$

Balanço de massa do produto $\boldsymbol{m}$ no CD $k$ :

$$
\sum_{j} \operatorname{Trns}_{m j k}+E I C D_{m k}=\sum_{l} D s t r_{m k l} \quad \forall m, k .
$$

Benefício do Drawback, que permite que um produto $\boldsymbol{m}$ exportado para o mercado $\boldsymbol{l}$, e que tenha sido fabricado na fábrica $\boldsymbol{i}$ no Brasil tenha isenção do II sobre a matéria prima:

$$
\sum_{i} \sum_{j} \operatorname{Trns}_{m i j} \leq \sum_{k} \sum_{l} \operatorname{Dstr}_{m k l} \quad \forall m, \quad i \in \text { Brazil }, \quad l \notin \text { Brazil }
$$

Variáveis binárias:

$$
\begin{aligned}
& Z_{j m}=\left\{\begin{array}{l}
1, \text { se } C D \text { jé usado para produto } m \\
0, \text { caso contrário }
\end{array} \quad \forall j, m\right. \\
& Z_{k}=\left\{\begin{array}{l}
1, \text { se } C D \text { ké usado } \\
0, \text { caso contrário }
\end{array} \forall k\right.
\end{aligned}
$$


Eliminação da geração de crédito de ICMS (balanço deve ser feito por estado $n$ ):

$$
\mathrm{VATB}_{n} \leq 0
$$

onde:

VATB $_{n}$ é o balanço de crédito de ICMS no estado $\boldsymbol{n}$ calculado como segue:

$$
V A T B_{n}=T C r_{n}-T D e b t_{n}-S D e b t_{n}
$$

em que,

$\mathrm{TCr}_{n}=$ Crédito de ICMS dos bens que serão recebidos nas instalações localizadas no estado $\boldsymbol{n}$

$$
=\sum_{m} \sum_{i} \sum_{j}\left(\operatorname{Trns}_{m i j}+\operatorname{Trns}_{m i j}\right) . P I_{m} \cdot I C M S_{i j}+\sum_{m} \sum_{j} \sum_{k} \operatorname{Trns}_{m j k} \cdot P I_{m} \cdot I C M S_{j k} \quad j, k \in \text { estado } n
$$

$\mathrm{TDebt}_{n}=$ Débito do ICMS dos itens que sairão de instalações localizadas no estado $\boldsymbol{n}$

$$
=\sum_{m} \sum_{j} \sum_{k} \operatorname{Trns}_{m j k} . P I_{m} \cdot \text { ICMS }_{j k} \quad j \in \text { estado } n
$$

SDebt $_{n}=$ Débito do ICMS dos itens que serão vendidos em CDs localizados no estado $\boldsymbol{n}$

$$
=\sum_{m} \sum_{k} \sum_{l} D s t r_{m k l} . P F_{m k l} \cdot I C M S_{k l} \quad k \in \text { estado } n
$$

$\mathrm{O}$ cálculo do custo de carregamento do estoque de segurança (CUSES) do produto $\boldsymbol{m}$ armazenado em $s$ CDs foi feito adaptando-se o modelo proposto por Croxton e Zinn (2005) mudando-se a variável $\boldsymbol{W}_{\boldsymbol{s}}$, onde o índice $\boldsymbol{c}$ refere-se à classe do produto acabado, para apenas $\boldsymbol{W}_{\boldsymbol{s}}$, já que, em nosso caso, a separação dos estoques de produto acabado por sua importância (classes A, B e C) não é relevante:

$$
\mathrm{CUSES}=\sum_{s} \sum_{m} I_{s m} W_{s}
$$


sujeito a:

$$
\begin{gathered}
\sum_{k} Z_{k}=\sum_{s} s W_{s} \\
\sum_{s} W_{s}=1
\end{gathered}
$$

onde:

$\boldsymbol{W}_{s}=$ Variável Binária e (25) e (26) determinam o número de CDs a serem abertos $\boldsymbol{I}_{\boldsymbol{s} \boldsymbol{m}}=$ Custo de Estoque do estoque de segurança de $\boldsymbol{m}$ em $\boldsymbol{s}$ CDs $\left(=S S_{s m} . P I_{m} . C F\right)$ $S S_{s m}$ foi calculado usando a clássica formulação proposta por Maister (1976):

$$
S S_{s m}=S S_{1 m} * \sqrt{S}
$$

onde:

$S S_{s m}=$ Estoque de segurança do produto $\boldsymbol{m}$ para $s$ armazéns $S S_{1 m}=$ Estoque de segurança do produto $\boldsymbol{m}$ para apenas um CD principal

Para calcular $S S_{1 m}$ foi usada a formulação proposta por Fetter, Darleck (1961) que considera todos os parâmetros importantes no cálculo de um estoque de segurança e que são perfeitamente aplicáveis nos casos estudados:

$$
S S_{l m}=\Phi_{m} *\left(\bar{t} \sigma_{x m}^{2}+\bar{x}_{m}^{2} \sigma_{t}^{2}\right)
$$

em que,

$\Phi_{m}=$ Número de vezes do desvio padrão da demanda dado nível de serviço desejado para $\boldsymbol{m}$

$\bar{t}=$ Lead-time médio em dias

$\sigma_{x m}=$ Desvio padrão da Demanda diária do produto $\boldsymbol{m}$ em dias

$\bar{x}_{m}=$ Demanda diária média do produto $\boldsymbol{m}$

$\sigma_{t}=$ Desvio padrão do lead-time de entrega em dias 


\section{Aplicação}

A abordagem aqui apresentada foi aplicada parcial ou integralmente a quatro casos reais de empresas transnacionais do setor químico que fornecem produtos com alto valor agregado para o mercado agrícola, com faturamento acima de US\$ 300 milhões apenas no Brasil. Todas as empresas que participaram deste estudo têm tido problemas para zerar seus créditos de ICMS e querem reduzir seus estoques, mas sem comprometer o nível de serviço oferecido. Em um caso, o problema a ser atacado era o consumo do crédito de ICMS. Em outro caso, o problema começou na definição de parceiros logísticos. Nos dois seguintes, o problema era a redução de custos. Em média, o trabalho de coleta, preparação de dados e adaptação do modelo em cada uma das empresas levou cerca de três meses.

A demanda por região não depende da localização das plantas industriais, mas pode ser levemente impactada pela localização dos CDs, por conta do lead-time de entrega. No caso das empresas estudadas, a aprovação de crédito e consequente liberação dos pedidos de venda apresentavam um tempo maior do que o tempo de coleta e trânsito e, portanto, foi adotada a premissa que a localização dos CDs não impactaria a demanda.

As empresas não autorizaram a apresentação dos dados de demanda, custo e capacidade usados neste estudo, por serem informações confidenciais. Todas elas não são empresas de capital aberto no Brasil e, portanto, preferem não divulgar essas informações para o mercado.

O modelo foi criado em MSExcel e usou o suplemento "Premium Solver" fornecido pela Frontline Systems, Inc. No cenário típico, o modelo contemplou 1297 variáveis (25 binárias) e 336 restrições mais as restrições de não-negatividade das variáveis. Um computador com um processador Intel® dual core e sistema operacional Microsoft Windows Vista foi usado. O tempo de processamento situou-se entre três e quatro segundos.

As quatro empresas que participaram deste estudo operam no setor do agronegócio, têm a sua sede em locais diferentes ao redor do mundo e todas elas têm ativos no Brasil há anos e importam total ou parcialmente o volume necessário de ingredientes ativos (matéria prima principal para a formulação de seus produtos).

Algumas outras semelhanças entre as empresas: 
- Nenhuma empresa escolheu investir em CDs secundários ou em sua própria frota;

- Todas trabalham com produtos de terceiros para revenda para complementar seu portfólio de soluções para o mercado agrícola;

- Todas tiveram iniciativas anteriores para reduzir o crédito podre, sem bons resultados;

- As iniciativas anteriores para reduzir o crédito podre foram capitaneadas pela área tributária com a participação de vários departamentos, mas o sucesso na implementação das ideias geradas foi pequeno;

- As decisões sobre mudanças na rede logística geralmente eram focadas na melhoria do nível de serviço;

- O projeto de revisão da rede logística com a abordagem aqui apresentada foi conduzido pelo Diretor de Supply Chain ou Diretor de Operações de cada empresa;

- O crédito de ICMS em carteira representava vários US\$ milhões;

- O preço unitário de venda era o mesmo para quaisquer regiões de vendas;

- As transferências entre CDs eram feitas usando-se o valor do CPV histórico;

- O foco maior era na otimização da rede logística, redução de custos e/ou geração de créditos de ICMS, mas era pré-requisito o não prejuízo ao nível de serviço;

- A revisão e liberação de ordens de venda para os clientes geralmente levava mais tempo do que os tempos de entrega, o que pressionava a área de logística no sentido da redução dos seus tempos de entrega.

Dentre as diferenças entre as empresas, vale a pena serem citadas:

- As fábricas estão localizadas em diferentes Estados do Brasil;

- Os principais fornecedores de ingredientes ativos estão em países diferentes;

- Apenas duas empresas vendem para outros países do Mercosul e apenas uma tem fábrica em outro país com licença para vender produtos no Brasil;

- Uma das empresas contabilizou prejuízos significativos no ano anterior ao estudo. 


\section{Resultados}

Por solicitação das empresas, os resultados numéricos não poderão ser apresentados em detalhes neste estudo. Mas todos eles foram validados pelas empresas e as ações sugeridas foram, na sua maioria, adotadas.

O custo de materiais em todas as empresas se mostrou o componente mais significativo no custo total ficando em torno de $90 \%$, típico de empresas químicas. O custo de carregamento de estoque, considerando um custo financeiro de $8 \%$, situou-se cerca da metade dos custos relacionados à soma dos fretes de distribuição e frete de transferência mais os custos de manuseio nos CDs e seguro.

O valor do crédito de ICMS sobre a projeção de vendas de um ano em relação ao custo total variou entre $1,2 \%$ e $5,1 \%$, mas o que chama a atenção é o crédito de ICMS existente em carteira em todos os casos, maior que a geração de crédito projetada para o ano. Em uma das empresas, o crédito acumulado ao longo dos anos chegava a dez vezes o crédito a ser gerado no ano do estudo conforme mostrado na Tabela 2.

Tabela 2: Representação dos valores de ICMS e custos parciais sobre o custo total

\begin{tabular}{|c|c|c|c|c|c|}
\cline { 2 - 6 } \multicolumn{1}{c|}{} & \multicolumn{2}{c|}{$\%$ ICMS } & \multicolumn{3}{c|}{$\%$ dos Custos } \\
\hline Empresa & Vendas & $\begin{array}{c}\text { Crédito } \\
\text { contábil }\end{array}$ & Materiais & $\begin{array}{c}\text { Frete Total + } \\
\text { Armazenagem }\end{array}$ & $\begin{array}{c}\text { Carregamento } \\
\text { do estoque }\end{array}$ \\
\hline 1 & $3,9 \%$ & $39,0 \%$ & $92,2 \%$ & $5,4 \%$ & $2,4 \%$ \\
\hline 2 & $5,1 \%$ & $5,2 \%$ & $87,3 \%$ & $7,8 \%$ & $4,9 \%$ \\
\hline 3 & $1,2 \%$ & $4,7 \%$ & $93,6 \%$ & $4,1 \%$ & $2,3 \%$ \\
\hline 4 & $2,4 \%$ & $2,9 \%$ & $93,3 \%$ & $4,2 \%$ & $2,5 \%$ \\
\hline
\end{tabular}

Fonte: Autores

A situação encontrada nas quatro empresas foi muito semelhante: 
- Taxas de importação atingiram em alguns casos mais de $10 \%$ do custo de materiais;

- Em geral, por ordem de importância dos custos logísticos, frete de distribuição ficou em primeiro lugar, seguido de perto pelos custos de estoque. Os custos de transferência e manuseio nos CDs teve um impacto menor, enquanto o custo fixo dos CDs e ad-valorem tiveram impacto mínimo;

- A fim de reduzir a saída de caixa e aproveitar os créditos de ICMS existentes, os custos de logística deveriam ser aumentados em praticamente todos os casos. Já os cenários que se preocupavam apenas com a minimização de custos levavam a piores fluxos de caixa;

- O custo de carregamento de estoque se mostrou muito maior do que os custos de frete de transferência, custos de manuseio e custo fixo dos CDs principais e secundários;

- A diferença de custo entre os cenários estudados e o cenário base chegou a US\$ 5 milhões em um dos casos (cerca de $3 \%$ do custo total);

- A diferença de fluxo de caixa em uma das empresas entre o cenário base e um dos cenários estudados atingiu o valor expressivo de US\$ 22 milhões (cerca de 5\% do custo total);

- Depois de um tempo sem uso, o crédito deve ser contabilizado como despesa por orientação de auditores e geralmente não pode ser provisionado. Esse impacto no resultado não previsto prejudicava a credibilidade do planejamento financeiro das filiais brasileiras perante a matriz;

- A utilização de CDs secundários revelou-se desejável em todos os casos justificada pela flexibilidade para a melhor gestão do ICMS, além da melhoria nos níveis de serviço;

- Em três das empresas, a instalação de uma fábrica em outro país do Mercosul para atender o mercado da região Sul do Brasil alimentado por ingredientes ativos vindos do exterior ou do Brasil e o fornecimento a partir do Brasil para esses mercados proporcionaria redução de custos interessantes, por eliminar a geração de créditos podres de ICMS e permitir a utilização dos benefícios do regime de Drawback/admissão temporária; 
- A análise de sensibilidade feita para avaliar o impacto do custo financeiro no número de CDs abertos usando um nível de serviço de $98 \%$ mostrou alta correlação entre esses fatores, mas pouca influência dos estoques de segurança (Figura 1)

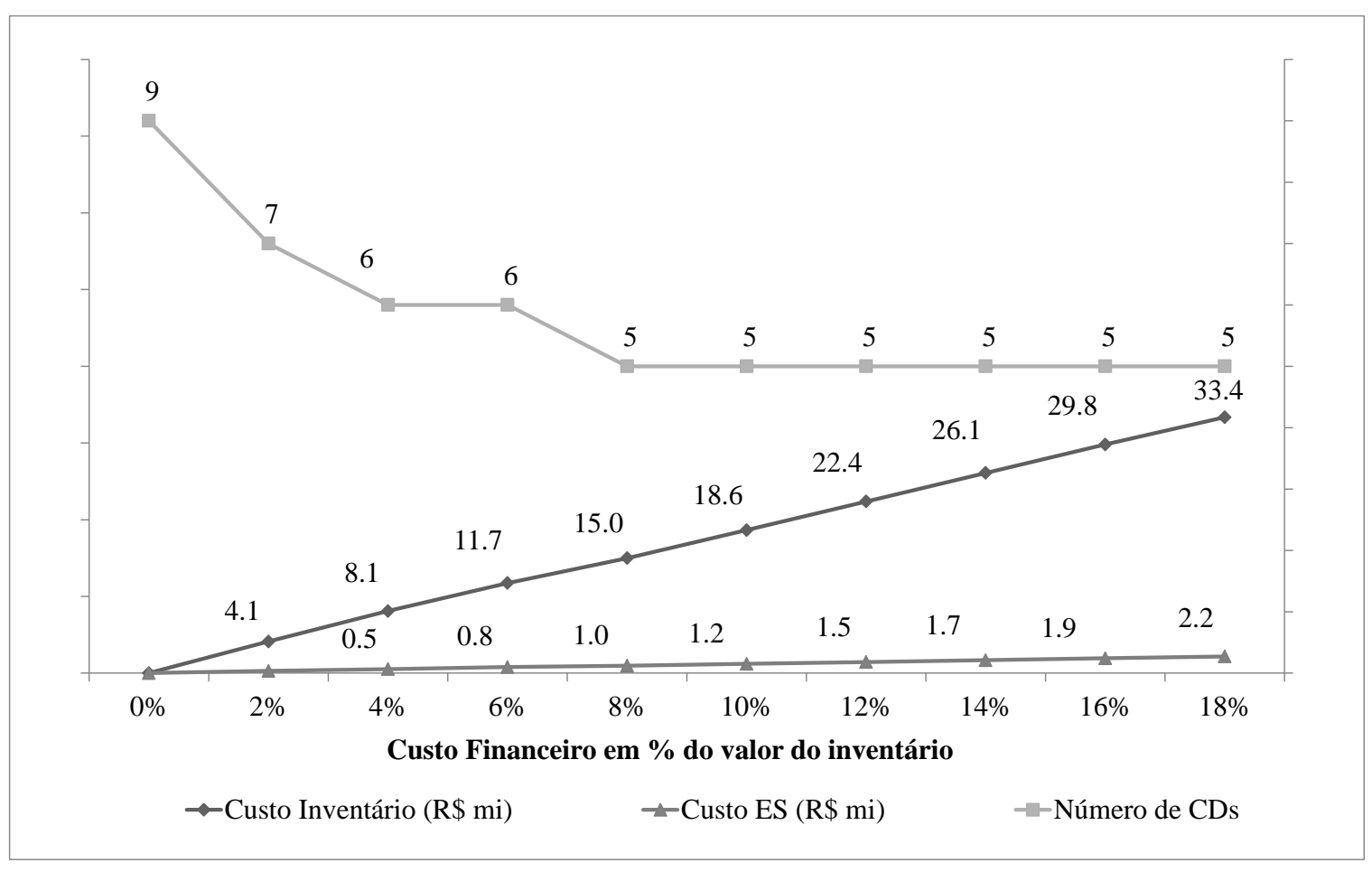

Figura 1 - Impacto do custo financeiro na quantidade de CDs abertos e a representatividade dos custos do Estoque de Segurança (ES)

- O número de CDs abertos é altamente influenciado pela cobertura esperada e pela capacidade de armazenagem dos CDs. Se os CDs funcionassem apenas para cross docking, um maior número de CDs se justificaria para melhor aproveitamento dos créditos de ICMS. Quando existe estoque, o número de CDs abertos se reduz por conta do custo de carregamento de estoque voltando a aumentar quando as capacidades de armazenagem são excedidas conforme mostra a Figura 2 para um custo de oportunidade financeira de $8 \%$. 


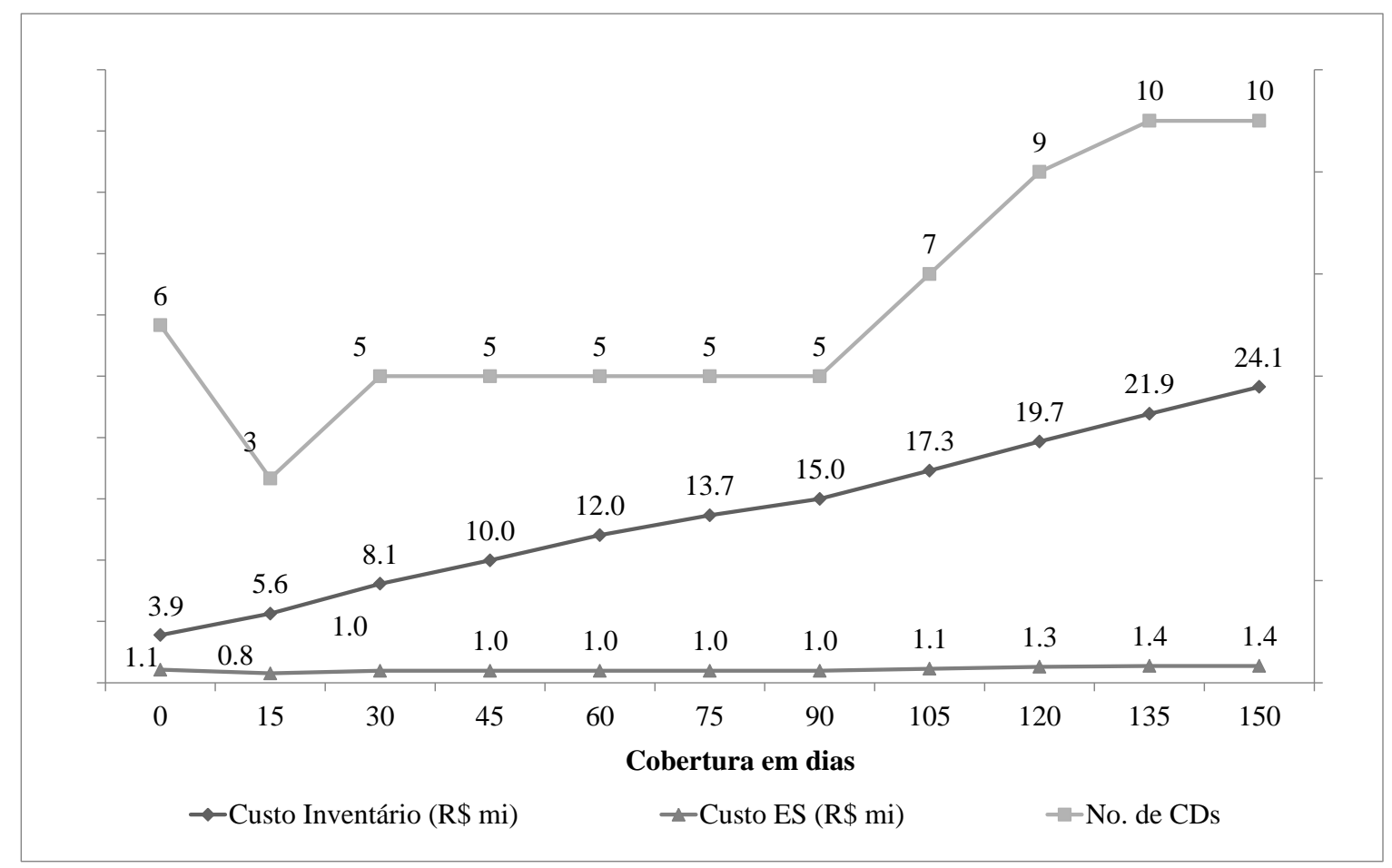

Figura 2 - Impacto da cobertura na quantidade de CDs abertos e a representatividade dos custos do Estoque de Segurança (ES)

- O valor de cobertura nas unidades industriais próprias variou entre 25 e 40 dias. Em uma análise de sensibilidade feita sobre o impacto dessa cobertura, demonstrou-se que, em média, se a necessidade de cobertura nas fábricas fosse o dobro, valeria mais a pena comprar os produtos acabados para revenda e não mais fabricá-los internamente;

- Durante o desenvolvimento dos estudos, percebeu-se que a modelagem com três elos seria insuficiente para a solução do problema estudado. E, apesar do modelo poder ser adaptado para cinco ou mais elos, quatro se mostraram suficientes para a solução do tipo de problema considerado. 


\section{Conclusões}

A metodologia apresentada mostrou-se adequada para resolver os diversos problemas apontados pelas empresas e o modelo matemático desenvolvido apresentou-se muito mais completo do que os encontrados na literatura por incorporar de forma integrada novidades como o tratamento dos créditos de ICMS e de custos de estoque, das restrições de armazenagem e do impacto do drawback. O grande impacto dos custos de carregamento do estoque total na definição da rede logística é mostrado de forma inequívoca e, portanto, estoques de matéria-prima e outros não podem ser desconsiderados. Foi provado também que o estoque de segurança não apresenta influência significativa nos resultados de definição da malha logística por representar uma fração dos estoques operacionais por conta da sazonalidade. Mesmo sabendo da importância do planejamento tributário para a gestão do fluxo de caixa e custos, as empresas têm dificuldades em usar essa ferramenta, devido à diversidade e complexidade da situação fiscal no Brasil e da quantidade de trade-offs envolvidos. A metodologia desenvolvida auxilia as empresas a atacar o problema de uma forma científica, sistêmica e mais estruturada.

As respostas dadas pela abordagem apresentada aqui para diferentes empresas transnacionais que operam no setor agrícola no Brasil atenderam plenamente as expectativas dessas empresas em termos de resultados e da aplicabilidade do modelo, da metodologia e das ações sugeridas. Embora as empresas estivessem geograficamente distribuídas em diferentes Estados e estavam em situação econômica muito diferente, as conclusões foram semelhantes. A diferença dos custos totais entre o caso base (situação inicial das empresas) e os resultados gerados pelo modelo mostrou oportunidades de redução de custo acima de US\$ 5 milhões. No que diz respeito a melhorias no fluxo de caixa, os resultados foram ainda mais significativos, da ordem de US\$ 13 milhões, graças à melhor utilização de créditos de ICMS, mas, para empresas com grandes volumes de crédito de ICMS, reduzir custos e melhorar o fluxo de caixa mostraram-se antagônicos no curto prazo, ao contrário do senso comum.

Os resultados confirmam a conclusão de outros autores sobre a importância dos tributos, principalmente ICMS e II, já que as estruturas destes impostos influenciam fortemente na definição de uma malha logística. A localização de instalações em outros países do Mercosul, por exemplo, se mostrou uma alternativa bastante vantajosa para a otimização do fluxo de 
caixa na região do Cone Sul, por causa do Drawback e gestão dos créditos de ICMS. No mercado brasileiro, CDs secundários distribuídos pelos estados são recomendáveis, porque melhoram o nível de serviço oferecido, reduzem os custos de transporte e permitem uma melhor gestão dos créditos de ICMS gerados.

Embora o modelo possa ser adaptado para cinco ou mais elos na cadeia, quatro elos provaram ser suficientes para resolver o problema de localização para a maioria das empresas. Para este tipo de problema, três elos não são suficientes.

Como limitação do modelo, destaca-se a assunção de que a localização dos CDs não impacta a demanda: No segmento agrícola a escolha do produto dá-se principalmente pela qualidade do produto, preço e suporte técnico, mas o tempo de entrega pode, em alguns casos, causar perda de venda o que precisa sem melhor explorado em melhorias do modelo ora apresentado.

Outra premissa é a de que todas as vendas serão atendidas. A perda da venda causa um custo que pode ser menor do que os custos de fabricação e logísticos necessários para se garantir a venda. $\mathrm{O}$ custo da perda de venda baseada na margem a ser perdida, nos custos de imagem, na perda do cliente, etc. poderiam ser incorporadas à função objetivo para que possa influenciar na decisão de localização.

\section{Referências}

Arntzen, B. C., Brown, G. G., Harrison, T. P. P. e Trafton, L. L. (1995) Global supply chain management at digital equipment corporation. Interfaces, vol. 25, n. 1, pp. 69-93

Associação Brasileira das Indústrias de Máquinas - ABIMAQ (2010) Impacto do "Custo Brasil" na competitividade da indústria brasileira. Texto não publicado. Disponível em www.abimaq.org.br.

Ballou, R. H. (1998) Business logistics management. 4e, Prentice-Hall, Englewood Cliffs, NJ

Bhutta, K. S. (2001) Global supply chains: an integration location, production, distribution e investment model for a multinational corporation operating under varying exchange rates and tariff structures. Doctoral Dissertation, University of Texas at Arlington

Bhutta, K. S. (2004) International facility location decisions: a review of the modeling literature. International journal of integrated supply management, vol. 1, n. 1, pp. 33-50

Bhutta, K. S., Huq, F., Frazier, G. e Mohamed, Z. (2003) An integration location, production, distribution and investment model for a multinational corporation. International Journal of Production Economics, vol. 86, n. 1, pp. 201-216

Bowersox, D. J. e Closs, D. J. (2001) Logística empresarial. O processo de integração da cadeia de suprimento. São Paulo, Atlas 
Canel, C. e Khumawala, B. M. (1996) A mixed integer programming approach for the international facilities location problem. International Journal of Operations and Production Management, vol. 16, No.4, pp. 49-68

Christopher, M. (2002) Logística e gerenciamento da cadeia de suprimentos - Estratégias para a redução de custos e melhoria dos serviços, Ed. Pioneira, São Paulo

Clark, A. J. (1958) A Dynamic, Single-Item/Multi-Echelon Inventory Model. RM2297, The Rand Corporation, Santa Monica, California

Croxton, K. L. e Zinn, W. (2005) Inventory considerations in network design. Journal of Business Logistics, vol. 26, n. 1, pp. 149 - 168

Dogan K. e Goetschalckx M. (1999) A primal decomposition method for the integrated design of multi-period production-distribution system. Research Report. IIE Transactions, vol. 31, No.11, pp.1027-1036

Erlenkotter, D. (1981) A comparative study of approaches to dynamic location problems. European Journal of Operational Research, vol. 6, pp. 133 - 143

Fetter, R.B. e Dalleck, W.C. (1961) Decision models for inventory management, Homewood, IL: Richard D. Irwin, Inc., pp. 105-108

Flipo, C. D. (2000) Spatial decomposition for a multi-facility production and distribution problem. International Journal of Production Economics, vol. 64, n. 1, pp. 177-186

Flipo, C. D. e Finke, G. (2001) An integrated model for an industrial production-distribution problem. IIE Transactions, vol. 33, n. 9, pp. 705-716

Garcia, E. S., Reis, L. M. T. V., Machado, L. R. e Ferreira, V. J. M. (2006) Gestão de estoques: otimizando a logística e a cadeia de suprimentos. Rio de Janeiro: E-papers Serviços Editoriais Ltda.

Goetschalckx, M., Cole, M. H., Dogan, K. e Wei, R. (1995) A generic model for the strategic design of production-distribution systems. Working Paper. Georgia Institute of Technology

Goetschalckx, M., Vidal, C. J. e Dogan, K. (2002) Modeling and design of global logistics systems: a review of integrated strategic and tactical models and design algorithms. European Journal of Operational Research, vol. 143, n. 1, pp. 1-18

Gross, D., Pinkus, C. E. e Soland, R. M. (1981) Designing a multi-product/multi-echelon inventory system. in 1.b. schwartz, "multi-commodity facilities location problem". Journal of the Operational Research Society, vol. 32, pp. 803-814

Hadjinicola, G. C. e Kumar, K. R. (2002) Modeling manufacturing and marketing options in international operations. International Journal of Production Economics, vol. 75, n. 1,pp. $287-$ 304

Hale, T. S., Day, J. M., Huq, F. e Pujari, N. A. (2008) A framework for an integrated distribution system optimisation model. International Journal of Logistics Systems and Management, Vol. 4, n. 5, pp. 506-522.

Hamad, R. e Gualda, N. D. F. (2006) Modelo para localização de instalações em escala global envolvendo quatro elos da cadeia logística. Transporte e Transformação, CNT, Brasília, vol. 10, pp. $59-73$

Hamad, R. e Gualda, N. D. F. (2008) Models for facilities or vendors location in a global scale considering several echelons in the chain. Networks and Spatial Economics, vol. 8, n. 1, pp. 297 $-307$ 
Hamad, R. e Gualda, N. D. F. (2011) Modelagem de redes logísticas com custos de inventário calculados a partir da cobertura de estoque. Revista Produção, São Paulo, Brasil, vol. 21, n. 4, pp. $667-675$

Harris, F. W. (1913) How Many Parts to Make at Once. Factory, The magazine of management, vol. 10, n. 2 , pp. $135-136,152$

Haug, P. P. (1985) A multiple-period, mixed-integer-programming model for multinational facility location. Journal of Management, vol. 11, n. 3, pp. 83-97

Hines, P. P. e Taylor, D. (2000) Going Lean. A guide to implementation. Lean Enterprise Research Center. Cardiff, UK.

Kirca, O. e Koksalan, M. (1996) An integrated production and financial planning model and an application. IIE Transactions, 19 (8), pp. $765-784$

Kuehn, A. A. e Hamburger, M. J. (1963) A Heuristic Program for Locating Warehouses. Management Science, vol. 9, pp. $643-666$

Love, R. F., Morris, J. G. e Wesolowsky, G. O. (1988) Facilities location: models \& methods. New York, North Holland, pp. 186-20

Machado, M. C. (2006) Princípios enxutos no processo de desenvolvimento de produtos: proposta de uma metodologia para implementação, Tese de doutorado, Universidade de São Paulo, Engenharia Politécnica, São Paulo.

Maister, D. H. (1976) Centralization of Inventories and the "Square Root Law". International Journal of Physical Distribution, vol. 6, n. 3, pp. 124 - 134.

Min, H. e Melachrinoudis, E. (1996) Dynamic location and entry mode selection of multinational manufacturing facilities under uncertainty: a chance-constrained goal programming approach. International Transactions of Operational Research, vol. 3, n. 1, pp. 6-76

Mohamed, Z. (1999) An Integrated Production-Distribution Model for a Multinational Company Operating Under Varying Exchange Rates. International Journal of Production Economics, vol. 58 , pp. $81-92$

Nozick, L. K. e Turnquist, M. A. (2001) Inventory transportation, service quality and the location of distribution centers. European Journal of Operations Research, vol. 129, n. 1, pp. 362-371

Owen, S. H. e Daskin, M. S. (1998) Strategic facility location: a review. European Journal of Operational Research, vol. 111, n. 1, pp. 423-447

PwC (2011) Sétima pesquisa de líderes empresariais brasileiros, crescimento reinventado. Texto não publicado. Disponível em www.pwc.com.

Revelle, C. S. e Eiselt, H. A. (2005) Location analysis: a synthesis and survey - invited review. European Journal of Operational Research, vol. 165, n. 1, pp. 1-19.

Richardson, H. (1995) Control your costs then cut them. Transportation \& Distribution.

Scarpati, K. (2011) Top 10 Supply chain concerns of 2011. Digital Supply Chain. Texto não publicado. Disponível em www.supplychaindigital.com.

Shapiro, J. F. (2001) Modeling the supply chain. Duxbury Press, Pacific Grove, CA.

Shen, Z. (2007) Integrated supply chain design models: a survey and future research directions. Journal of Industrial and Management Optimization, vol. 3, n. 1, pp. 1 - 27.

Shen, Z., Coullard, C. e Daskin, M. S. (2003) A joint location-inventory model. Transportation Science, vol. 37, n. 1, pp. 40-55. 
Smits, S. R. (2001) Evaluation model for the design of distribution networks. Faculty of technology management, Eindhoven, The Netherlands

Snyder, L. V. (2006) Facility location under uncertainty: a review, IIE Transactions, 38, pp. 537-554.

Syam, S. S. (2002) A model and methodologies for the location problem with logistical components. Computers and Operations Research, V 29, n. 1, pp. 1173 - 1193

Tomich, F. A., Magalhães, L. C. G., Guedes, E. M. e Carvalho, A. X. Y. (1998), Desoneração do ICMS da cesta básica, planejamento e políticas públicas, vol. 17, pp. 213-253

Verter, V. (2002) An integrated model for facility location and technology acquisition. Computers and Operations Research, vol. 29, pp. 583-592

Vidal, C. J. e Goetschalckx, M. (1997) Strategic production-distribution models: A critical review with emphasis on global supply chain models. European Journal of Operational Research, vol. 98, n. 1, pp.1-18.

Weber, A. (1909) Uber den standort der industrien (alfred weber's theory of the location of industries), University of Chicago

Wilson, R. H. (1934) A scientific routine for stock control. Harvard Business Review, vol. 13, pp. 116128

Womack, J. P., Jones, D. T. e Roos, D. (1990) The machine that changed the world, Rawson Associates, New York, USA.

Yoshizaki, H. T. Y. (2002) Projeto de redes de distribuição física considerando a influência do ICMS, Tese de Livre-Docência, Universidade de São Paulo, Escola Politécnica da USP, São Paulo. 